\title{
A Class of Nonconvex Nonsmooth Approximate Potential Functions for Nonconvex Nonsmooth Image Restoration
}

\author{
Xiao-Guang Liu \\ College of Mathematics and Information Science \\ Shaanxi Normal University \\ Xi'an, Shaanxi \\ liuxiaoguang_lxg@163.com
}

\author{
Xing-Bao Gao \\ College of Mathematics and Information Science \\ Shaanxi Normal University \\ Xi'an, Shaanxi \\ xingbaog@snnu.edu.cn
}

\begin{abstract}
Nonconvex nonsmooth potential functions have superior restoration performance for the images with neat boundaries. However, several difficulties emerge from the numerical computation. Thus the graduated nonconvex (GNC) method is suggested to deal with these problems. To improve the performance of the GNC method further, a class of nonconvex nonsmooth approximate potential functions have been constructed in this paper, which can help our get a better initial value of the original problem. The numerical results show the restored quality and efficiency of the proposed methods.
\end{abstract}

Keywords-nonconvex nonsmooth; potential function; image restoration; algorithm

\section{INTRODUCTION}

Digital image restoration has a wide application in various areas including Navigation, Biomedicine and so on [1-2]. In general, the relationship between the original image $f \in R^{l}$ and the observed image $g \in R^{q}$ is:

$$
g=H f+b,
$$

where the spatial-invariant matrix $H \in R^{q \times l}$ represents the degradation systems caused by problems such as motion blur and distortion radiation. $l=m \times n$ ( $m$ and $n$ represent the number of rows and columns respectively when image is expressed as a matrix). $b \in R^{q}$ is the additive noise.

The aim of the image restoration is to obtain an estimate of the original image $f$ according to some knowledge about $H, g$, and $b$. However it often tends to be very illconditioned when the reverse process of the model (1) is only used to get ideal solution $\hat{f}$. Thus one of the effective ways to solve the problem is to combine some priori information of the original image and define the regularization solution, i.e., $\hat{f}$ is a minimum point of the following cost function (energy function) :

$$
J(f)=\theta(H f-g)+\beta \Phi(f),
$$

where $\theta: R^{q} \rightarrow R$ is a measure of the difference between Hf and $g$. $\Phi$ embodies the priori information, and a regularization parameter $\beta>0$ is used to control the tradeoff between the terms $\theta$ and $\Phi$.
In general, we set the additive noise meets Gaussian distribution, then $\theta(x)=\|x\|_{2}^{2}$. The regularization term $\Phi(f)=\sum_{i \in I} \phi\left(\left\|D_{i} f\right\|_{2}\right)$, where $I=\{1,2, \cdots, l\}, \phi: R \rightarrow R_{+}$is a potential function with $R_{+}=\{t \in R: t \geq 0\}$. The matrix $D_{i}: R^{l} \rightarrow R^{s}$ is the difference operator which can be viewed as a $s \times l$ matrix and used to create the difference vector between $i$ th pixel and its $s$ neighboring pixels. Generally, $s$ is selected as 2 .

In the image restoration, potential function $\phi$ plays a key role so that it is intensively studied[3-11]. For the images with neat boundaries, nonconvex nonsmooth potential functions have superior restoration performance from the theories and numerical experiments, but it also causes several difficulties in numerical computation[6-8]. For example, gradient-based methods are inappropriate, global minimum point can not be attained. Thus the graduated nonconvex (GNC) method[9-11] is widely employed. Even though the global convergence of this method could not be guaranteed, the experiment results show that the energy in (2) is lower than many famous methods such as simulated annealing and so on[12-13]. However, most nonconvex smooth approximate potential functions can track the performance of the nonconvex smooth potential function perfectly, but they could not do very well when potential function is nonconvex nonsmooth. So a class of nonconvex nonsmooth approximate potential functions is introduced in [10-11] and the good restored performance is obtained.

To improve the performance of the GNC method further when the potential function is nonconvex nonsmooth, in this paper, we construct a new class of nonconvex nonsmooth approximate potential functions $\phi_{\varepsilon_{k}}$ whose similarity between $\phi_{\varepsilon_{k}}$ and $\phi$, as well as $\phi_{\varepsilon_{k}}$ and prior approximate potential function $\phi_{\varepsilon_{k-1}}$ could be easily controlled, specially for the given $\varepsilon_{k}$. The numerical results in section III will indicate the performance.

In the following discussions, we let $R^{+}=\{t \in R \mid t \neq 0\}$, $R_{+}^{*}=\{t \in R \mid t>0\}, \operatorname{Her}(H)=\left\{x \in R^{l} \mid H x=0\right\}$. For the convenience of later discussions, the lemma below is introduced to summarize the basic properties of $\phi$ and $\phi_{\varepsilon_{k}}$. 
Lemma $1^{[11]}$ Let $\phi_{\varepsilon_{N}}=\phi$, if potential function $\phi$ and approximate potential function $\phi_{\varepsilon_{k}}(k=1,2, \cdots, N)$ satisfy:

1) $\operatorname{Ker}(H) \cap \operatorname{Ker}(D)=0$, where $D=\left[D_{1}^{\mathrm{T}}, D_{2}^{\mathrm{T}}, \cdots, D_{l}^{\mathrm{T}}\right]^{\mathrm{T}}$;

2) $\phi(t)$ is continuous and symmetric on $R$ and increasing on $R_{+}, \phi(0)=0$ and $\phi^{\prime}\left(0^{+}\right)>0, \lim _{t \rightarrow \infty} \phi^{\prime \prime}(t)=0$;

3) $\phi(t) \in C^{2} \quad\left(\quad R_{+}^{*} / M \quad\right)$, where $M=\left\{t \in R^{+} \mid \phi^{\prime}\left(t^{-}\right)>\phi^{\prime}\left(t^{+}\right)\right.$and $\left.-\infty<\phi^{\prime \prime}\left(t^{-}\right)<\phi^{\prime \prime}\left(t^{+}\right) \leq 0\right\}$ is a continuous set of points(possibly empty);

4) $\phi^{\prime \prime}\left(0^{+}\right)<0, \phi^{\prime \prime}(t) \leq 0$ and increasing on $R_{+}^{*} / M$;

5) Both $\phi^{\prime}\left(0^{+}\right)>0$ and $\phi^{\prime \prime}\left(0^{+}\right)<0$ are finite, $\phi_{\varepsilon_{0}}(t)=\phi^{\prime}\left(0^{+}\right)|t|$;

6) for each $\varepsilon_{k} \in[0,1], \phi_{\varepsilon_{k}}(t)$ is continuous and symmetric on $R, \phi_{\varepsilon_{k}}(t) \in C^{2}\left(R_{+}^{*}\right)$-smooth, $\phi_{\varepsilon_{k}}(0)=0, \phi_{\varepsilon_{k}}^{\prime \prime}(t)<0$ on $R^{+}$, both $\phi_{\varepsilon_{k}}^{\prime}\left(0^{+}\right)>0$ and $\phi_{\varepsilon_{k}}^{\prime \prime}\left(0^{+}\right)<0$ are finite.

Then $\varphi_{\varepsilon_{k}}(t)=\phi_{\varepsilon_{k}}(t)+\phi_{\varepsilon_{k}}^{\prime}\left(0^{+}\right)|t|$ satisfies:

1) $\varphi_{\varepsilon_{0}}(t)=0, \varphi_{\varepsilon_{1}}(t)=\phi(t)-\phi^{\prime}\left(0^{+}\right)|t|$ for all $t \in R$;

2) $\varphi_{\varepsilon_{k}}^{\prime}(0)=0, \varphi_{\varepsilon_{0}}(t) \in C^{2}$ and $\varphi_{\varepsilon_{k}}^{\prime \prime}(-t)=\varphi_{\varepsilon_{k}}^{\prime \prime}(t)<0$ on $R$.

\section{The New Nonconvex}

\section{Nonsmooth APPROXIMATE POTENTIAL FUNCTION}

In this section, we will propose a new class of approximate potential functions with perfect performance, specially, for the given $\varepsilon_{k}$.

When $\phi$ is nonconvex nonsmooth, a series of approximate potential functions $\phi_{\varepsilon_{k}}$ are constructed to approach $\phi$ gradually so that a good initial value can be obtained in GNC method. It is worth noting the restoration performance will not be stable enough, when the difference between $\phi_{\varepsilon_{k}}$ and $\phi$ is too large or small, as well as the difference between $\phi_{\varepsilon_{k}}$ and $\phi_{\varepsilon_{k-1}}(k \geq 1)$, so the similarity between $\phi_{\varepsilon_{k}}$ and $\phi, \phi_{\varepsilon_{k}}$ and $\phi_{\varepsilon_{k-1}}$ are very important. In this paper, firstly, we select the following potential function as example:

$$
\phi(t)=\frac{\alpha|t|}{1+\alpha|t|},
$$

then the nonconvex nonsmooth approximate potential function $\phi_{\varepsilon_{k}}^{\text {old }}$ in [11] is:

$$
\phi_{\varepsilon_{k}}^{\text {old }}(t)=\frac{\alpha|t|}{1+\varepsilon_{k} \alpha|t|} .
$$

We can know from [11] that it has a good performance since the similarity between $\phi_{\varepsilon_{k}}$ and $\phi, \phi_{\varepsilon_{k}}$ and $\phi_{\varepsilon_{k-1}}$ can be controlled by different $n$. However it is hard to change the similarity for the given $\varepsilon_{k}$.

To overcome this drawback of $\phi_{\varepsilon_{k}}^{\text {old }}$, we consider the approximate potential function:

$$
\phi_{\varepsilon_{k}}^{\text {new }}(t)=\frac{\alpha|t|\left(c|t| \varepsilon_{k}+1\right)}{1+c|t| \varepsilon_{k}+\left(c|t|+\varepsilon_{k}\right) \varepsilon_{k} \alpha|t|},
$$

where $c>0$ is a constant. Obviously, $\phi_{\varepsilon_{k}}^{\text {new }}$ is nonconvex and nonsmooth, $\phi_{\varepsilon_{k}}^{\text {new }}(0)=\phi(0), \lim _{t \rightarrow \infty} \phi_{\varepsilon_{k}}^{\text {new }}(t)=\lim _{t \rightarrow \infty} \phi(t)$, and $\phi_{\varepsilon_{n}}^{\text {new }}=\phi$, then the similarity between $\phi_{\varepsilon_{n}}^{\text {new }}$ and $\phi$ is guaranteed. More importantly, constant $c$ can be used to control the similarity between $\phi_{\varepsilon_{k}}^{\text {new }}$ and $\phi, \phi_{\varepsilon_{k}}^{\text {new }}$ and $\phi_{\varepsilon_{k-1}}^{\text {new }}$ for the given $\varepsilon_{k}$. To see this clearly, Figure 1 (a) and (b) are drawn to illustrate the similarity between $\phi_{\varepsilon_{k}}^{\text {new }}$ and $\phi$, $\phi_{\varepsilon_{k}}^{\text {new }}$ and $\phi_{\varepsilon_{k-1}}^{\text {new }}$ how to be influenced by the parameter $c$ respectively. From Figure 1, we see that the parameter $c$ can not only control flexibly the similarity between $\phi_{\varepsilon_{k}}^{\text {new }}$ and $\phi$, as well as $\phi_{\varepsilon_{k}}^{\text {new }}$ and $\phi_{\varepsilon_{k-1}}^{\text {new }}$ in the neighborhood of the origin for the given $\varepsilon_{k}$, but also keep the nonconvexity nonsmoothness. This is helpful for us to get more effective restoration (the intensity often be set from 0 to 1 ).
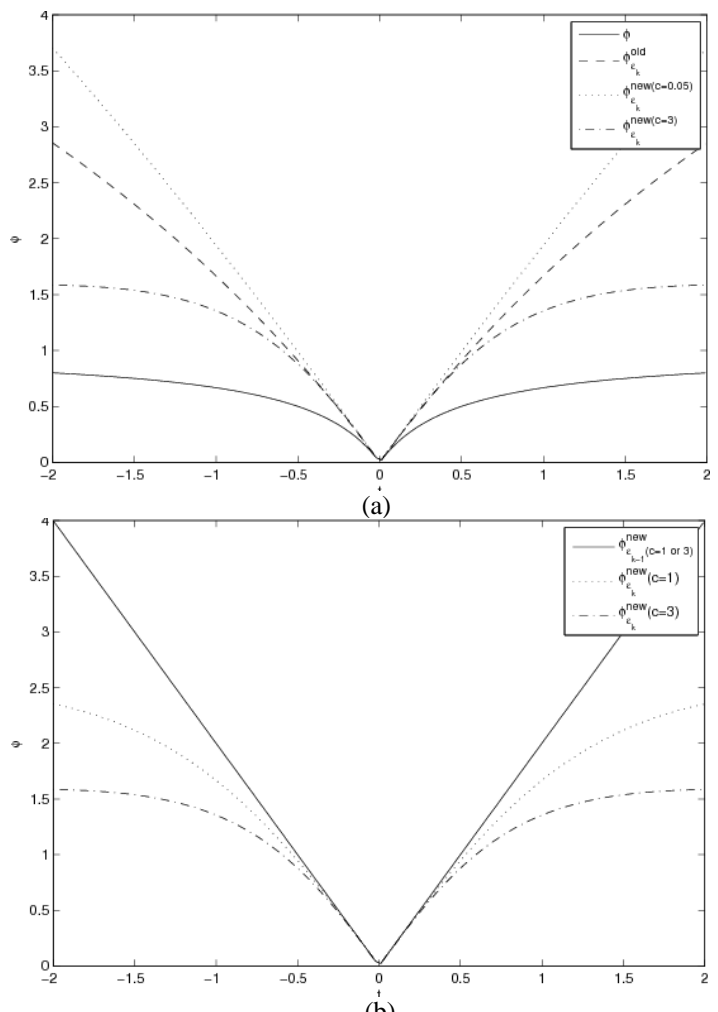

(b)

Fig. 1. New approximate potential functions: (a) $\alpha=2, \varepsilon_{k}=0.1$, and different $c$. (b) $\alpha=2, \varepsilon_{k}=0.1, \varepsilon_{k-1}=0$ and different $c$.

To show the performance of $\phi_{\varepsilon_{k}}^{\text {new }}$ in (5) further and for the convenience of later discussions, the numerical results based 
on the two algorithms in [11](denoted by algorithm I and algorithm II) are reported in subsection III. From these numerical results, we see that $\phi_{\varepsilon_{k}}^{\text {new }}$ in (5) has perfect restoration performance over $\phi_{\varepsilon_{k}}^{\text {old }}$.

Remark 1. In general, $\phi$ can also be chosen as:

$$
|t|^{\alpha}(0<\alpha<1), \quad \log (\alpha|t|+1)
$$

and more choices can be found in [10-11], new approxin potential functions for them can be defined similarly.

\section{EXPERIMENTAL RESULTS}

In this section, numerical experiment for two tested images will be provided to show the performance of $\phi_{i}$ All computations are performed using MATLAB2010a v Core(TM)2CPU with $2.83 \mathrm{GHz}$ and 3.87GB of RAM. Si the noise is a gaussian distribution, we report the average value of ten tests.

The tested images are UTK and Cameraman(CR). $\phi$, । and $\phi_{\varepsilon_{k}}^{\text {new }}$ are defined as (3)-(5) respectively. CPU tim used to compare the efficiency of the restoration, and the quality of the restored images is measured by Peak signalto-noise ratio (PSNR):

$P S N R=-20 \log _{10}\left(\frac{\left\|f^{\left(j, \varepsilon_{k}\right)}-f\right\|_{2}}{m n}\right)$,

It is easy to verify that $\phi_{\varepsilon_{k}}^{\text {new }}$ satisfies the conditions of

Lemma 1. Two algorithms in [11] will be used to show the performance of them. As in [11], we set absolute error(Abs) is $10^{-4}$, max iterations is 1000 , the regularization parameter $\beta=0.015, \alpha=0.5$. The initial value of $\omega$ is 0.05 , and its value is updated by $1.8 \omega$ at each inner iteration, $n=20$. The step size used in the Chambolle's method is 0.25 . The tested blurring function is chosen to be truncated 2-D Gaussian function:

$h(s, t)=\exp \left(\frac{-s^{2}-t^{2}}{2 \sigma^{2}}\right),-3 \leq s, t \leq 3$,

where three sets of parameters are chosen as: (1) $\sigma=1$, the support is $7 \times 7$, and the standard deviation of noise is 0.05 ; (2) $\sigma=1.5$, the support is $9 \times 9$, and the standard deviation of noise is 0.1 ; (3) $\sigma=2$, the support is $11 \times 11$, and the standard deviation of noise is 0.2 . To illustrate the stability of the parameter $c$, it is selected as 0.02 and 0.03 respectively.

Figures 2-3(a) show the original images. Figures 2-3(b) show the observed images. Figures 2(c) shows the restored UTK image by the algorithm I in [11] with $\phi_{\varepsilon_{k}}^{\text {old }}$, Figures 2(d) shows the restored UTK image by the algorithm I in [11] with $\phi_{\varepsilon_{k}}^{\text {new }}$, where the standard deviation of noise is 0.1 , the support is $9 \times 9$, and $c=0.02$. Figures 3 (c) shows the restored CR image by the algorithm II the method in [11] with $\phi_{\varepsilon_{k}}^{\text {old }}$, Figures $3(\mathrm{~d})$ shows the restored CR image by the algorithm II in [11] with $\phi_{\varepsilon_{k}}^{\text {new }}$, where the standard deviation of noise is 0.2 , the support is $11 \times 11$, and $c=0.03$. With different $c$, more restoration results will be summarized in Table 1-4.
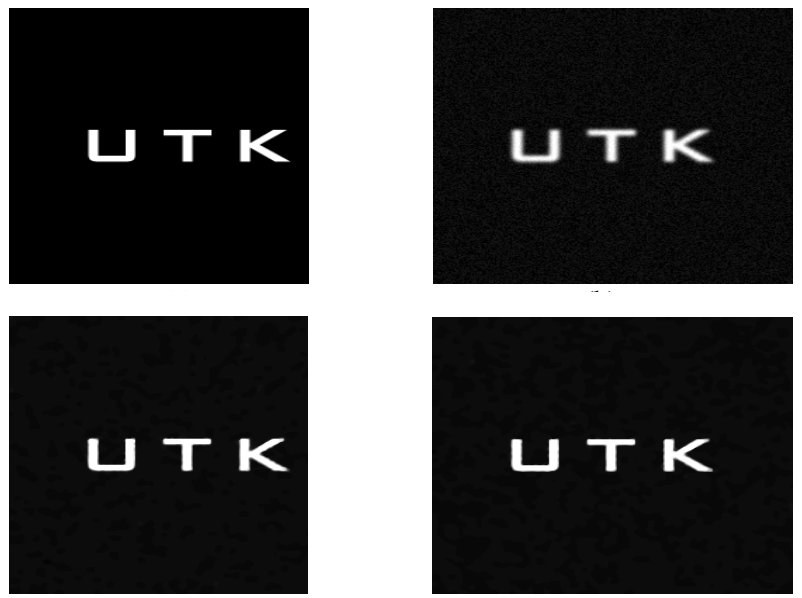

(c)

Fig. 2. The restored UTK images by algorithm I with different approximate potential functions, the standard deviation of noise is 0.1 , the support is $9 \times 9, c=0.02$. (a) Original image. (b) Observed image. (c) Image restored by algorithm I in [11] with $\phi_{\varepsilon_{k}}^{\text {old }}$. (d) Image restored by algorithm I in [11] with $\phi_{\varepsilon_{k}}^{\text {new }}$.

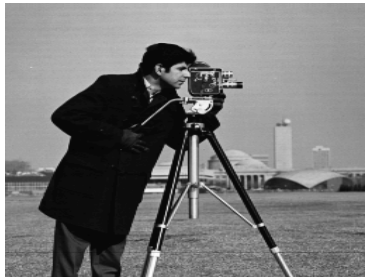

(a)

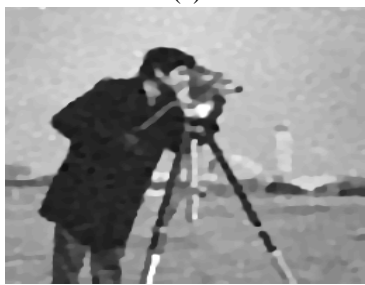

(c)

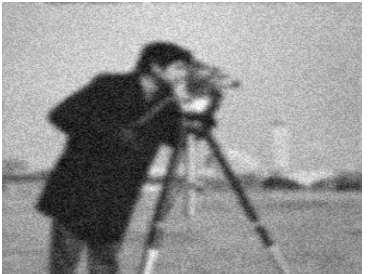

(b)

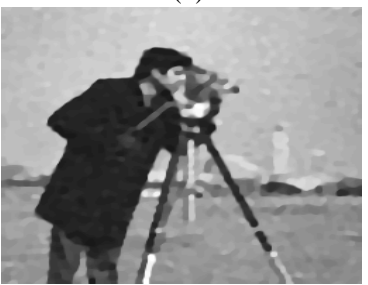

(d)
Fig. 3. The restored CR images by algorithm I with different approximate potential functions, the standard deviation of noise is 0.2 , the support is $11 \times 11, c=0.03$. (a) Original image. (b) Observed image. (c) Image restored by algorithm II in [11] with $\phi_{\varepsilon_{k}}^{\text {old }}$. (d) Image restored by algorithm II in [11] with $\phi_{\varepsilon_{k}}^{\text {new }}$.

TABLE 1 Algorithm I with $\phi_{\varepsilon_{k}}^{\text {old }}$ and $\phi_{\varepsilon_{k}}^{\text {new }}$ when $C=0.02$

\begin{tabular}{|c|l|c|c|c|c|c|c|}
\hline \multirow{2}{*}{} & \multicolumn{2}{|c|}{} & observed & \multicolumn{2}{c|}{ old } & \multicolumn{2}{|c|}{ new } \\
\cline { 2 - 8 } & NOISE & BLUR & PSNR & PSNR & CPU & PSNR & CPU \\
\hline \multirow{2}{*}{ C } & 0.05 & 7 & 24.74 & 26.22 & 5.10 & 26.56 & 4.32 \\
\cline { 2 - 8 } & 0.1 & 9 & 21.21 & 22.64 & 7.52 & 22.77 & 5.50 \\
\hline
\end{tabular}




\begin{tabular}{|c|l|c|c|c|c|c|c|}
\cline { 2 - 8 } $\mathrm{R}$ & 0.2 & 11 & 17.21 & 18.44 & 8.41 & 18.46 & 6.84 \\
\hline $\mathrm{U}$ & 0.05 & 7 & 27.85 & 31.73 & 10.87 & 31.88 & 8.01 \\
\cline { 2 - 8 } $\mathrm{T}$ & 0.1 & 9 & 23.09 & 25.73 & 17.58 & 26.06 & 14.99 \\
\cline { 2 - 8 } $\mathrm{K}$ & 0.2 & 11 & 18.05 & 18.79 & 23.03 & 18.82 & 16.03 \\
\hline
\end{tabular}

TABLE 2 Algorithm II with $\phi_{\varepsilon_{k}}^{\text {old }}$ and $\phi_{\varepsilon_{k}}^{\text {new }}$ when $C=0.02$

\begin{tabular}{|c|l|c|c|c|c|c|c|}
\hline & & & observed & \multicolumn{2}{|c|}{ old } & \multicolumn{2}{|c|}{ new } \\
\cline { 2 - 9 } & NOISE & BLUR & PSNR & PSNR & CPU & PSNR & CPU \\
\hline C & 0.05 & 7 & 24.74 & 26.22 & 2.17 & 26.22 & 1.72 \\
\cline { 2 - 8 } & 0.1 & 9 & 21.20 & 22.59 & 2.34 & 22.63 & 1.90 \\
\cline { 2 - 8 } R & 0.2 & 11 & 17.21 & 18.43 & 2.26 & 18.39 & 2.20 \\
\hline U & 0.05 & 7 & 27.84 & 31.74 & 4.20 & 31.80 & 4.13 \\
\cline { 2 - 8 } T & 0.1 & 9 & 23.07 & 25.74 & 4.34 & 25.77 & 3.91 \\
\cline { 2 - 8 } K & 0.2 & 11 & 18.04 & 19.76 & 4.27 & 19.77 & 4.05 \\
\hline
\end{tabular}

TABLE 3 Algorithm I with $\phi_{\varepsilon_{k}}^{\text {old }}$ and $\phi_{\varepsilon_{k}}^{\text {new }}$ when $c=0.03$

\begin{tabular}{|c|l|c|c|c|c|c|c|}
\hline \multirow{2}{*}{} & \multicolumn{2}{|c|}{} & observed & \multicolumn{2}{|c|}{ old } & \multicolumn{2}{|c|}{ new } \\
\cline { 2 - 9 } & NOISE & BLUR & PSNR & PSNR & CPU & PSNR & CPU \\
\hline \multirow{2}{*}{$\mathrm{C}$} & 0.05 & 7 & 24.72 & 26.22 & 5.10 & 26.56 & 4.32 \\
\cline { 2 - 9 } & 0.1 & 9 & 21.22 & 22.64 & 7.52 & 22.67 & 5.50 \\
\cline { 2 - 8 } $\mathrm{R}$ & 0.2 & 11 & 17.21 & 18.43 & 8.41 & 18.78 & 6.84 \\
\hline $\mathrm{U}$ & 0.05 & 7 & 27.86 & 31.73 & 10.87 & 32.01 & 8.01 \\
\cline { 2 - 8 } $\mathrm{T}$ & 0.1 & 9 & 23.08 & 25.70 & 17.58 & 25.73 & 14.99 \\
\cline { 2 - 8 } $\mathrm{K}$ & 0.2 & 11 & 18.06 & 18.79 & 23.03 & 18.82 & 19.03 \\
\hline
\end{tabular}

TABLE 4 Algorithm II with $\phi_{\varepsilon_{k}}^{\text {old }}$ and $\phi_{\varepsilon_{k}}^{\text {new }}$ when $c=0.03$

\begin{tabular}{|c|l|c|c|c|c|c|c|}
\hline \multirow{2}{*}{} & \multicolumn{2}{|c|}{} & observed & \multicolumn{2}{|c|}{ old } & \multicolumn{2}{|c|}{ new } \\
\cline { 2 - 9 } & NOISE & BLUR & PSNR & PSNR & CPU & PSNR & CPU \\
\hline C & 0.05 & 7 & 24.73 & 26.33 & 2.16 & 26.52 & 1.90 \\
\cline { 2 - 9 } & 0.1 & 9 & 21.22 & 22.69 & 2.50 & 22.67 & 2.01 \\
\cline { 2 - 8 } R & 0.2 & 11 & 17.21 & 18.42 & 2.31 & 18.33 & 1.74 \\
\hline U & 0.05 & 7 & 27.84 & 31.63 & 4.22 & 31.71 & 4.01 \\
\cline { 2 - 8 } $\mathrm{T}$ & 0.1 & 9 & 23.08 & 25.77 & 4.31 & 25.83 & 3.99 \\
\cline { 2 - 8 } $\mathrm{K}$ & 0.2 & 11 & 18.04 & 18.79 & 4.27 & 18.92 & 3.63 \\
\hline
\end{tabular}

From Fig. 2-3, we see that we can get the better restored images by algorithms I and II in [11] with $\phi_{\varepsilon_{k}}^{\text {new }}$ in most cases. Tables 1-2 report the restored PSNR and CPU times for algorithm I and algorithm II in [11] with $\phi_{\varepsilon_{k}}^{\text {old }}$ and $\phi_{\varepsilon_{k}}^{\text {new }}$ respectively, when $c=0.02$. Tables 3-4 report the restored PSNR and CPU times for algorithm I and algorithm II in [11] with $\phi_{\varepsilon_{k}}^{\text {old }}$ and $\phi_{\varepsilon_{k}}^{\text {new }}$ respectively, when $c=0.03$. We could see that that algorithm I and algorithm II in [11] with $\phi_{\varepsilon_{k}}^{\text {new }}$ is faster than the algorithm I and algorithm II in [11] with $\phi_{\varepsilon_{k}}^{\text {old }}$ in any case. The restored quality has been improved in most cases.

\section{CONCLUDING REMARS}

In this paper, we propose one kind of approximate potential function to improve the performance of the GNC method further in image restoration. Extended numerical experiments have been given to illustrate the restored qualities and efficiencies. It has been shown that the restored quality is litter better than the methods in.[11], but the efficiency is significantly higher in any case.

\section{REFERENCES}

[1] M. R. Banham and A. K. Katsaggelos, Digital image restoration, IEEE Trans. Signal Process., vol.14, pp.24-41, 1997.

[2] R. C. Gonzalez and R. E. Woods, Digital image processing, second ed, Prentice Hall, New Jersey, 2002.

[3] X. X. Guo, F. Li, and M. K. Ng, A fast $l_{1}$-TV algorithm for image restoration, SIAM J. Sci. Comput., vol.31, pp.2322-2341, 2009.

[4] H. Y. Fu, M. K. Ng, M. Nikolova, and J. L. Barlow, Efficient minimization methods of mixed $l_{2}-l_{1}$ and $l_{1}-l_{1}$ norms for image restoration, SIAM J. Sci. Comput., vol.27, pp.1881-1902, 2006.

[5] A. M. Bruckstein, D. L. Donoho and M. Elad, From sparse solutions of systems of equations to sparse modeling of signals and images, SIAM Rev., vol.51, pp. 34-81, 2009.

[6] K. C. Kiwiel, Convergence of the gradient sampling algorithm for nonsmooth nonconvex optimization, SIAM J. Optim., vol.18, pp. 379-388, 2007.

[7] F. H. Clarke, Optimization and Nonsmooth Analysis, John Wiley and Sons, New York, 1983.

[8] J. V. Burke, D. Henrion, A. S. Lewis and M. L. Overton, Stabilizationvia nonsmooth, nonconvex optimization, IEEE Trans. Automat. Contr., vol.51, pp.1760-1769, 2006.

[9] L. Bedini, I. Gerace, and A. Tonazzini, A GNC algorithm forconstrained image reconstruction with continuous-value line process, Pattern Recognit, Lett., vol.15, pp.907-918, 1994.

[10] M. Nikolova, Markovian reconstruction using a GNC approach, IEEE Trans. Image Process., vol.8, pp.1204-1220, 1999.

[11] M. Nikolova, M. K. Ng and C. P. Tam, Fast Nonconvex Nonsmooth Minimization Methods for Image Restoration and Reconstruction, IEEE Tran. Image Process., vol.19, pp. 3073-3088, 2010.

[12] S. Kirkpatrick, C. D. Gelatt, and M. P. Vecchi, Optimization by simulated annealing, Science, vol.220, pp.671-680, 1983.

[13] S. Boyd and L. Vandenberghe, Convex Optimization, Cambridge University, Cambridge, 2004.

[14] A. Chambolle, An algorithm for total variation minimization and applications, J. Math. Imag. Vis., vol.20, pp. 89-97, 2004. 